\title{
Plasma particle sources due to interactions with neutrals in a turbulent scrape-off layer of a toroidally confined plasma
}

Thrysøe, Alexander Simon; Løiten, M.; Madsen, J.; Naulin, Volker; Nielsen, A. H.; Rasmussen, J. Juul

Published in:

Physics of Plasmas

Link to article, DOI:

$10.1063 / 1.5019662$

Publication date:

2018

Document Version

Publisher's PDF, also known as Version of record

Link back to DTU Orbit

Citation (APA):

Thrysøe, A. S., Løiten, M., Madsen, J., Naulin, V., Nielsen, A. H., \& Rasmussen, J. J. (2018). Plasma particle sources due to interactions with neutrals in a turbulent scrape-off layer of a toroidally confined plasma. Physics of Plasmas, 25(3), [032307]. https://doi.org/10.1063/1.5019662

\section{General rights}

Copyright and moral rights for the publications made accessible in the public portal are retained by the authors and/or other copyright owners and it is a condition of accessing publications that users recognise and abide by the legal requirements associated with these rights.

- Users may download and print one copy of any publication from the public portal for the purpose of private study or research.

- You may not further distribute the material or use it for any profit-making activity or commercial gain

- You may freely distribute the URL identifying the publication in the public portal 


\section{Plasma particle sources due to interactions with neutrals in a turbulent scrape-off layer of a toroidally confined plasma}

A. S. Thrysøe, M. Løiten, J. Madsen, V. Naulin, A. H. Nielsen, and J. Juul Rasmussen

Citation: Physics of Plasmas 25, 032307 (2018); doi: 10.1063/1.5019662

View online: https://doi.org/10.1063/1.5019662

View Table of Contents: http://aip.scitation.org/toc/php/25/3

Published by the American Institute of Physics

\section{Articles you may be interested in}

Gyrokinetic theory for particle and energy transport in fusion plasmas

Physics of Plasmas 25, 032306 (2018); 10.1063/1.5018175

Transition between resistive ballooning mode and toroidal drift wave mode at the edge of tokamak plasmas Physics of Plasmas 25, 032505 (2018); 10.1063/1.5009680

Three-dimensional simulations of plasma turbulence in the RFX-mod scrape-off layer and comparison with experimental measurements

Physics of Plasmas 25, 022305 (2018); 10.1063/1.5008803

Modelling of nitrogen seeding experiments in the ASDEX Upgrade tokamak

Physics of Plasmas 25, 032506 (2018); 10.1063/1.5019913

Two-dimensional turbulence cross-correlation functions in the edge of NSTX

Physics of Plasmas 24, 102509 (2017); 10.1063/1.5002695

The collisionality dependence of intermittency level in drift-wave turbulence in the stellarator TJ-K

Physics of Plasmas 24, 112307 (2017); 10.1063/1.4991609

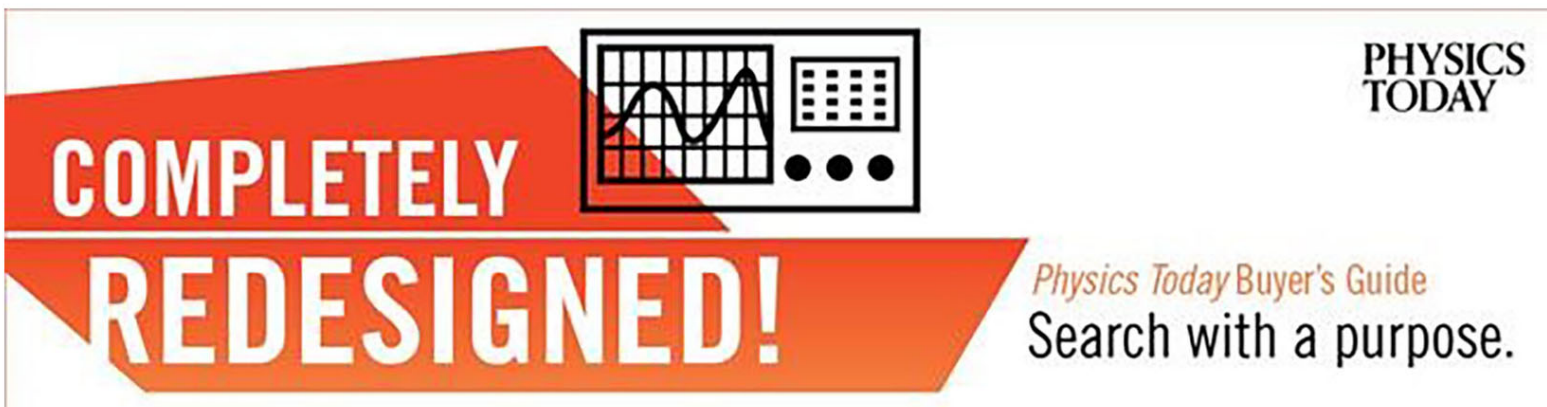




\title{
Plasma particle sources due to interactions with neutrals in a turbulent scrape-off layer of a toroidally confined plasma
}

\author{
A. S. Thrysøe, ${ }^{\text {a) }}$ M. Løiten, J. Madsen, V. Naulin, A. H. Nielsen, and J. Juul Rasmussen \\ PPFE, Department of Physics, DTU, DK-2800 Kgs, Lyngby, Denmark
}

(Received 15 December 2017; accepted 28 February 2018; published online 20 March 2018)

\begin{abstract}
The conditions in the edge and scrape-off layer (SOL) of magnetically confined plasmas determine the overall performance of the device, and it is of great importance to study and understand the mechanics that drive transport in those regions. If a significant amount of neutral molecules and atoms is present in the edge and SOL regions, those will influence the plasma parameters and thus the plasma confinement. In this paper, it is displayed how neutrals, described by a fluid model, introduce source terms in a plasma drift-fluid model due to inelastic collisions. The resulting source terms are included in a four-field drift-fluid model, and it is shown how an increasing neutral particle density in the edge and SOL regions influences the plasma particle transport across the lastclosed-flux-surface. It is found that an appropriate gas puffing rate allows for the edge density in the simulation to be self-consistently maintained due to ionization of neutrals in the confined region. https://doi.org/10.1063/1.5019662
\end{abstract}

\section{INTRODUCTION}

One of the main drivers for determining the plasma confinement in magnetically confinement devices such as tokamaks is the dynamics in the edge region of the bulk plasma. This region is characterized by the transition from closed to open magnetic field lines, in which the last-closed-flux-surface (LCFS) defines the boundary between the confined plasma and the scrape-off layer (SOL), where the field lines terminate on material surfaces. The anomalous transport of particles and energy across the LCFS is strongly intermittent, and it has been found that most transport is carried by fieldaligned filaments (blobs) that are formed near the plasma edge and propagate radially outwards towards the first wall. ${ }^{1-4}$

When the plasma filaments interact with the material surfaces, the plasma undergoes surface recombination, and neutral atoms and molecules are released through various mechanisms of which most result in low-energy neutrals. ${ }^{5}$ This process is referred to as plasma recycling. Neutral particles can also originate from being injected into the vacuum vessel for fuelling or diagnostic purposes. ${ }^{6,7}$ Typical SOL temperatures that are much lower than those of the bulk plasma allow for a significant population of neutrals to exist. The neutral atoms and molecules will interact with the plasma both through elastic and inelastic collisions. Elastic collisions between charged particles and neutrals are not treated in this paper, but the effect of such collisions in driftfluid models are derived in Ref. 8. The inelastic collisions are, for example, excitation and ionization of atoms and molecules and dissociation of molecules. The ionization process, if inside the confined region, is what fuels the plasma. Ionization may also occur in the SOL and prevent the neutrals from fuelling the plasma directly. In addition to their fuelling properties, neutrals may also provide protection to

\footnotetext{
${ }^{\text {a)} E l e c t r o n i c ~ m a i l: ~ a l e c @ f y s i k . d t u . d k . ~}$
}

the first wall from the plasma filaments by cooling down the plasma through elastic and inelastic collisions.

There exist a number of numerical models for simulating the neutral-plasma interactions. Both kinetic neutral models such as in Refs. 9-12 and fluid models ${ }^{13-16}$ have previously been applied to study SOL and edge plasmas in the presence of neutrals. In this paper, we derive a neutral fluid model for the purpose of studying the effects of neutrals on the intermittent plasma fields. A fluid model allows for a dynamical treatment of the neutrals and thus selfconsistently simulates the response of SOL plasma structures to neutrals and vice versa. This model in particular takes into account the long mean-free path of neutral atoms in the SOL region, which prevents the usual Chapman-Enskog closure ${ }^{17}$ from being applied to the neutral fluid equations.

The source terms resulting from inelastic neutral-plasma interactions are introduced in a plasma drift-fluid model. Drift-fluid models are derived from the two-fluid equations and describe the physics of phenomena that occur on timescales much slower than the ion cyclotron frequency and length scales much larger than the ion gyroradius. This results in a simpler fluid model which is still able to describe the low frequency turbulence in the edge and SOL regions such as interchange-driven turbulence. In this paper, we demonstrate that the presence of inelastic density and momentum sources introduce additional drift-terms, and the resulting source terms are included in an existing drift-fluid based plasma model.

This paper has two main sections: In Sec. II, the HESEL $^{18,19}$ drift-fluid model is presented (Sec. II A), and it is displayed how inelastic neutral source terms enter such a model (Sec. IIC). It is also described how neutrals with a long mean-free path between collisions, for which the usual Chapman-Enskog closure scheme is no longer valid, can be described in a fluid model (Sec. II B). A combined plasmaneutral model is given in Sec. II D, where the equations for the HESEL drift-fluid model are enhanced with neutral source terms. The combined HESEL-neutral model is solved 
self-consistently, and the results are discussed in Sec. III. In this section, it is shown how the presence of neutrals affects the SOL density and temperature profiles, when gas puffing is simulated at three different puffing rates (Sec. III A). Moreover, we display in Sec. III B how fuelling in HESEL can be self-consistently described in the combined neutralplasma model, when the electron/ion density source in the inner edge region is replaced by a source solely from ionization of neutrals. The latter will allow for future studies on the effect of gas puffing on edge plasmas and thus the effect of gas puffing on overall confinement.

\section{MODELS FOR TRANSPORT AND INTERACTIONS}

In this section, we present two models. The first model is a four-field drift-fluid based model for plasma variables. Drift-fluid models originate from the Braginskii fluid equations where an ordering in powers of the ion gyration frequency in the momentum equations is introduced. This technique is known as drift-ordering, and it reduces the number of variables described by eliminating the momentum equation by describing the electron and ion fluid velocities as a sum of drift-fluid velocities. The technique is described in detail in Refs. 20 and 21. The particular drift-fluid model considered in this paper is the HESEL model, and it describes the evolution of the electron/ion density, the generalized vorticity, and the electron and ion pressures. The ultimate purpose of this section is to augment an existing driftfluid model with source terms originating from interactions with neutrals. The neutrals are described as multiple fluids to take into account both neutral molecules and atoms at distinct characteristic temperatures. The neutrals interact selfconsistently with the plasma variables, so that the effect of the inelastic collisions results in source and sink terms in both the neutral and plasma transport equations.

The section is structured so that Sec. II A provides the HESEL model for the case with no source terms from neutral interactions. In Sec. II B, the derivation and validity of a neutral fluid model are discussed. Section IIC displays how source terms enter the plasma drift-fluid equations, and in Sec. II D, a combined plasma-neutral model is proposed.

\section{A. HESEL equations}

The HESEL model ${ }^{18,19}$ describes the transport of density, ion and electron pressures, and generalized vorticity at the outboard-midplane of a tokamak in a drift-plane perpendicular to the magnetic field lines. The original HESEL model describes the evolution of plasma variables without the presence of neutral particles. Here, we couple the HESEL model and a neutral model described in Secs. II B and IIC. Since HESEL describes both the electron and ion temperature dynamics, the influence of neutral particles on both species can be studied in edge and SOL plasmas. The inclusion of neutrals also allows for the density source in the original HESEL equations to be partially replaced with one that self-consistently originates from ionization of neutral particles. The results of including neutrals in a plasma turbulence model are given in Sec. III.
The original HESEL equations without neutral source terms are derived from the drift-fluid equations and read

$$
\begin{gathered}
\mathrm{d}_{t} n+n \mathcal{K}(\phi)-\mathcal{K}\left(p_{\mathrm{e}}\right)=\Lambda_{n}, \\
\nabla \cdot\left(\mathrm{d}_{t}^{0} \nabla_{\perp} \phi^{*}\right)-\mathcal{K}\left(p_{\mathrm{e}}+p_{\mathrm{i}}\right)=\Lambda_{\omega}, \\
\frac{3}{2} \mathrm{~d}_{t} p_{\mathrm{e}}+\frac{5}{2} p_{e} \mathcal{K}(\phi)-\frac{5}{2} \mathcal{K}\left(p_{\mathrm{e}}^{2} / n\right)=\Lambda_{p_{\mathrm{e}}}, \\
\frac{3}{2} \mathrm{~d}_{t} p_{\mathrm{i}}+\frac{5}{2} p_{i} \mathcal{K}(\phi)+\frac{5}{2} \mathcal{K}\left(p_{\mathrm{i}}^{2} / n\right)-p_{\mathrm{i}} \mathcal{K}\left(p_{\mathrm{e}}+p_{\mathrm{i}}\right)=\Lambda_{p_{\mathrm{i}}},
\end{gathered}
$$

where $n$ is the electron/ion density, $\phi$ is the potential, $p_{\mathrm{e}, \mathrm{i}}$ $=n T_{\mathrm{e}, \mathrm{i}}$ are the electron and ion pressures, $\phi^{*}=\phi+p_{\mathrm{i}}$ is the modified potential, and the $\Lambda$ terms on the RHS contain the terms from collisional diffusion and terms for parametrized loss along the magnetic field lines and are discussed in detail in Refs. 18 and 19. The advective derivatives are defined as $\mathrm{d}_{t}=\partial_{t}+B^{-1}\{\phi, \cdot\}$ and $\mathrm{d}_{t}^{0}=\partial_{t}+\{\phi, \cdot\}$, with the antisymmetric bracket $\{f, g\}=\partial_{x} f \partial_{y} g-\partial_{y} f \partial_{x} g$. The variables are gyro-Bohm normalized, so that

$$
\begin{array}{lll}
\Omega_{\mathrm{ci}} t \rightarrow t, & \frac{x}{\rho_{s}} \rightarrow x, & \\
\frac{e \phi}{T_{\mathrm{e} 0}} \rightarrow \phi, & \frac{T_{\mathrm{e}, \mathrm{i}}}{n_{0}} \rightarrow n, & T_{\mathrm{e} 0} T_{\mathrm{e}, \mathrm{i}} .
\end{array}
$$

with $\Omega_{\mathrm{ci}}$ being the ion cyclotron frequency, $\rho_{\mathrm{s}}=\sqrt{\frac{T_{\mathrm{e} 0}}{m_{\mathrm{i}} \Omega_{\mathrm{ci}}^{2}}}$ the cold-ion hybrid thermal gyro-radius, $T_{\mathrm{e} 0}$ a characteristic electron temperature, and $n_{0}$ a characteristic density, and we have introduced the curvature operator

$$
\mathcal{K}=-\frac{\rho_{\mathrm{s}}}{R} \partial_{y}
$$

where $R$ is the major radius of the tokamak.

\section{B. Neutral transport model}

When deriving a combined neutral-plasma model, in which both neutrals and plasma are treated dynamically, it is computationally meaningful to also seek out a fluid model for the neutral particles. A neutral fluid model can be derived the same way as the plasma fluid model, i.e., by taking velocity moments of the kinetic equation but without including the terms involving electric and magnetic fields. Deriving the neutral fluid equations in this manner is a classical textbook example (see, for example, Ref. 22), in which the set of equations is usually asymptotically closed in the Chapman-Enskog scheme which leads to a set of equations similar to the Braginskii equations but again without the electric and magnetic field terms. The Chapman-Enskog closure is valid for a gas where the particles experience a meanfree path between collisions which is short compared to the characteristic length scales in the system. In the following, we examine the validity of this assumption for neutral particles in a tokamak.

In a tokamak, hydrogen isotope molecules can either be puffed into the vacuum chamber for the purpose of fuelling or perturbing the plasma or result from recombination of atoms on the first wall. Reaction rates for ion-molecule 
inelastic collisions are in general low for SOL-relevant temperatures, but electrons interact with molecules in a number of ways. Electron impact collisions can lead to excited states of the molecules, ionize the molecules one or two times, or dissociate the molecule to atoms and/or ions, depending on whether the molecule was ionized before dissociation.

A dissociated molecule results in warm $2 \mathrm{eV}$ neutral atoms ${ }^{23}$ known as Franck-Condon neutrals. Warmer neutral atoms have a perpendicular mean-free path much longer than that of ions since they are not influenced by the magnetic field, and the atoms may obtain energies even higher than $2 \mathrm{eV}$ from charge-exchange collisions with ions. Energetic neutrals can also originate from recombination of plasma on the first wall. For SOL parameters, the mean-free paths $\lambda$ of typical neutral particles are ${ }^{16}$

$$
\lambda=\frac{v_{\mathrm{th}, \mathrm{n}}}{\nu} \approx \frac{\sqrt{T_{\mathrm{n}} / m_{\mathrm{n}}}}{\langle\sigma v\rangle n} \approx \begin{cases}10^{-2} \mathrm{~m} & \text { for } T_{\mathrm{n}}=25 \mathrm{meV} \\ 10^{0} \mathrm{~m} & \text { for } T_{\mathrm{n}}=2 \mathrm{eV} \\ 10^{1} \mathrm{~m} & \text { for } T_{\mathrm{n}}=50 \mathrm{eV}\end{cases}
$$

where $50 \mathrm{eV}$ represents a neutral atom resulting from a charge-exchange collision with a hot ion. $v_{\mathrm{th}, \mathrm{n}}$ is the thermal speed, $\nu=\langle\sigma v\rangle n$ is the collision frequency, $T_{\mathrm{n}}$ is the typical energy of neutral species $\mathrm{n}$, and $m_{\mathrm{n}}$ is the mass. In reality, the neutrals have a continuous spread in the energy around the thermal energy, but the above comparison gives an idea of the relevant length scales.

The conclusion is that thermal neutral molecules have a mean-free path comparable to that of the gradient length scales of SOL structures, whereas hotter neutral atoms have mean-free paths much longer than those. This poses a problem for a fluid description of the neutral atoms since the conventional Chapman-Enskog closure ${ }^{17}$ is not valid in this regime.

Neutral fluid descriptions that extend beyond the assumption of a short mean-free path are sparsely treated in the literature. It is, however, investigated in Ref. 24 when seeking a fluid description for the electron particle and heat transport in the direction of magnetic field lines in a low collisionality tokamak plasma, which also experiences a long mean-free path. This paper finds that a fluid closure is generally not obtainable in terms of profiles in the long mean-free path limit, but surprisingly the particle transport is well described, with the density flux given by Fick's law. For the neutral particle case, this results in a continuity equation of the form

$$
\partial_{t} n_{\mathrm{n}}-\nabla \cdot\left(D_{\mathrm{n}} \nabla n_{\mathrm{n}}\right)=S_{\mathrm{n}}^{n}
$$

with

$$
D_{\mathrm{n}}=\frac{1}{2} \frac{v_{\mathrm{th}, \mathrm{n}}^{2}}{\nu},
$$

for any collisionality $\nu$. Here, $n_{\mathrm{n}}$ is the density of neutral species $\mathrm{n}$ and $S_{\mathrm{n}}^{n}$ is the corresponding density source.

Since a heat equation on closed form is not obtainable in this regime, the neutral temperatures have to be accounted for in other manners. We propose a model that resolves the neutral velocity distribution to a finite number of isothermal neutral species, each with its own diffusion coefficient determined by the energy of that species. The main driver for such assumption is rooted in the atomic physics described above; the neutral molecules enter at thermal velocity, and the neutral atoms are created at distinct temperatures. The energy exchange rate between neutrals is low compared to their average lifetime, and they are thus assumed to maintain their initial energy until ionization. The assumption is supported experimentally, where distinct highly populated energies in the neutral velocity spectrum have also been observed, both from spectroscopic measurements of the Zeeman-split Balmer $\operatorname{lines}^{25}$ and from laser-induced fluorescence ${ }^{26}$ on the TEXTOR tokamak. The experimental findings report slightly lower typical energies for the dissociated neutrals than what is assumed here, most likely due to the dissociation into atoms in the $n=3$ state, instead of the ground state.

The assumptions made by describing the neutral populations at distinct temperatures may, however, lead to an oversimplification of the physical processes that could be essential for describing observed phenomena. In particular, effects of having neutrals in a broader energy range originating from recycling and from charge exchange collisions are neglected, as the recycled neutrals are not included in the analysis presented here, and those originating from charge exchange are all assigned the same temperature. Despite this, resolving the neutral velocity distribution into three temperatures is assumed to reproduce the primary effects of a kinetic description. The characteristic decay lengths for the neutral densities are taken into account, which allows for quantifying the spatial influence of the different types of neutrals on the edge and SOL plasma.

In the model presented here, we describe the neutral atoms and molecules by three species corresponding to those with temperatures given in (7), i.e., cold neutral molecules, warm neutral atoms originating from dissociated molecules, and hot neutral atoms from charge-exchange collisions between warm neutrals and ions.

The transport equations are coupled to each other and to those of the plasma variables through inelastic collisions. The interactions with the highest cross-section in the SOL and edge domains, and that describe the neutral particle life cycle outlined in the beginning of the section, are included. The corresponding source terms enter the neutral particle transport equations (8) with $\mathrm{n}=$ cold, warm, and hot as

$$
\begin{aligned}
S_{\text {cold }}^{n}= & -n_{\mathrm{e}} n_{\text {cold }}\left(\left\langle\sigma_{\text {Dis }} v\right\rangle+\left\langle\sigma_{\mathrm{Iz}} v\right\rangle\right), \\
S_{\text {warm }}^{n}= & n_{\mathrm{e}}\left(n_{\text {cold }}\left(2\left\langle\sigma_{\text {Dis }} v\right\rangle+\left\langle\sigma_{\mathrm{Iz}} v\right\rangle\right)\right. \\
& \left.-n_{\text {warm }}\left\langle\sigma_{\mathrm{iz}} v\right\rangle\right)-n_{\mathrm{i}} n_{\mathrm{warm}}\left\langle\sigma_{\mathrm{cx}} v\right\rangle, \\
S_{\text {hot }}^{n}= & n_{\mathrm{i}} n_{\mathrm{warm}}\left\langle\sigma_{\mathrm{cx}} v\right\rangle-n_{\mathrm{e}} n_{\text {hot }}\left\langle\sigma_{\mathrm{iz}} v\right\rangle,
\end{aligned}
$$

where $n_{\text {cold }}$ is the density of the molecules and $n_{\text {warm }}$ and $n_{\text {hot }}$ are those for the atomic species.

The above set of source terms accounts for dissociation (Dis) of molecules (e $+\mathrm{H}_{2} \rightarrow \mathrm{e}+2 \mathrm{H}$ ), ionization followed by dissociation (Iz) of molecules $\left(\mathrm{e}+\mathrm{H}_{2} \rightarrow 2 \mathrm{e}+\mathrm{H}_{2}^{+}\right.$ $\rightarrow 2 \mathrm{e}+\mathrm{H}^{+}+\mathrm{H}$ ), ionization (iz) of atoms (e $+\mathrm{H} \rightarrow$ $2 \mathrm{e}+\mathrm{H}^{+}$), and charge-exchange collisions (cx) between warm atoms and hot ions $\left(\mathrm{H}^{+}+\mathrm{H} \rightarrow \mathrm{H}+\mathrm{H}^{+}\right)$. $\mathrm{H}$ can be any hydrogen isotope, as the reaction rates only depend weakly on the 
isotope mass. Those are among the dominant reaction rates in the temperature interval of interest. Other reactions could be included as well, such as charge exchange collisions between ions and molecules or dissociation of molecules into excited atomic states. Including those reactions evidently results in the same end-products as are covered by the present model, but the location and rate of the creation of atoms and ions from molecules may change. The effects of including those reactions are left for future work. The reaction rates as a function of energy are shown in Fig. 1.

This concludes the neutral model, and the effect of the interactions on the plasma drift-fluid equations are described in Sec. II C.

\section{Plasma-neutral interactions}

The presence of inelastic neutral interactions such as ionization of atoms introduces source terms to the transport equations. Formally, the Boltzmann equation becomes

$$
\partial_{t} f_{\sigma}+\boldsymbol{v} \cdot \nabla f_{\sigma}+\frac{q_{\sigma}}{m_{\sigma}}(\boldsymbol{E}+\boldsymbol{v} \times \boldsymbol{B}) \cdot \nabla_{\boldsymbol{v}} f_{\sigma}=\mathcal{C}_{\sigma}+\mathcal{S}_{\sigma},
$$

where an inelastic source (or sink) term $\mathcal{S}$ is introduced on the RHS. Velocity space moments of (13) result in a set of fluid equations that differ from the Braginskii two-fluid equations by the appearance of the RHS source terms

$$
\begin{gathered}
\partial_{t, \sigma} n_{\sigma}+\cdots=S_{\sigma}^{n}, \\
m_{\sigma} n_{\sigma} \partial_{t, \sigma} \boldsymbol{u}_{\sigma}+\cdots=\boldsymbol{S}_{\sigma}^{u}, \\
\frac{3}{2} \partial_{t, \sigma} p_{\sigma}+\cdots=S_{\sigma}^{p},
\end{gathered}
$$

where the source terms are formally obtained by taking moments of $\mathcal{S}_{\sigma}$ from (13) as discussed in Ref. 13.

For the electron impact molecular dissociation, molecular ionization and dissociation, atomic ionization, and ionatom charge exchange, the above source terms take the form

$$
S^{n}=S^{\mathrm{iz}}+S^{\mathrm{Iz}}=n_{\mathrm{e}}\left[\left(n_{\mathrm{warm}}+n_{\mathrm{hot}}\right)\left\langle\sigma_{\mathrm{iz}} v\right\rangle+n_{\text {cold }}\left\langle\sigma_{\mathrm{Iz}} v\right\rangle\right]
$$

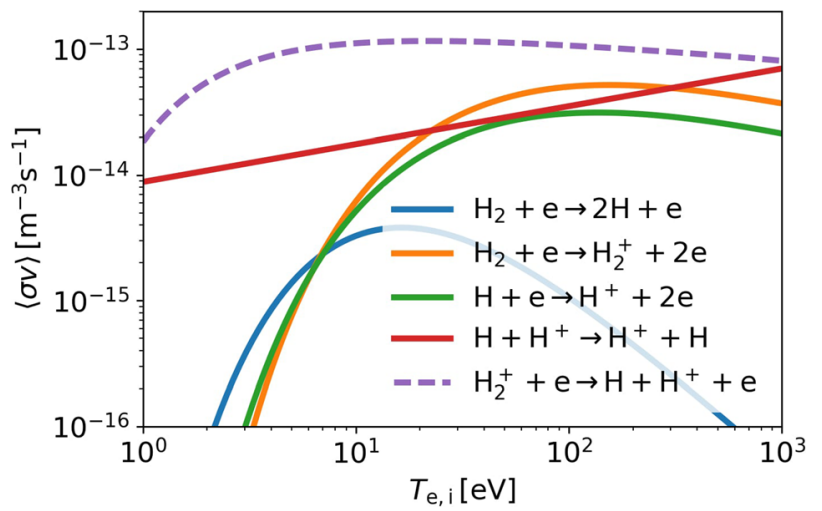

FIG. 1. Dominant reaction rates for inelastic collisions between ions or electrons and neutrals. The molecular assisted ionization is a two-step reaction, where the reaction rate for the dissociation of $\mathrm{H}_{2}^{+}$is shown by the dashed curve. Due to the high reaction rate at all relevant temperatures and the short mean-free path of $\mathrm{H}_{2}^{+}$compared to that of neutrals, this reaction is assumed to happen immediately after ionization of $\mathrm{H}_{2}$. The reaction rates are obtained from the study by Janev et al. ${ }^{27}$ for the density source,

$$
\begin{gathered}
\boldsymbol{S}_{\mathrm{e}}^{u}=m_{\mathrm{e}}\left(\boldsymbol{u}_{\mathrm{n}}-\boldsymbol{u}_{\mathrm{e}}\right) S^{n}, \\
\boldsymbol{S}_{\mathrm{i}}^{u}=m_{\mathrm{i}}\left(\boldsymbol{u}_{\mathrm{n}}-\boldsymbol{u}_{\mathrm{i}}\right)\left(S^{n}+S^{\mathrm{cx}}\right)+\boldsymbol{R}_{\mathrm{in}}^{\mathrm{cx}}-\boldsymbol{R}_{\mathrm{ni}}^{\mathrm{cx}},
\end{gathered}
$$

for the momentum sources, and the energy density sources are

$$
\begin{aligned}
S_{\mathrm{e}}^{p}= & \frac{3}{2} \frac{m_{\mathrm{e}}}{m_{\mathrm{n}}} T_{\mathrm{n}} S^{n}+\frac{1}{2} m_{\mathrm{e}}\left(\boldsymbol{u}_{n}-\boldsymbol{u}_{\mathrm{e}}\right)^{2} S^{n}+\frac{1}{2} m_{\mathrm{e}} u_{\mathrm{e}}^{2} S^{n} \\
& -\phi_{\mathrm{iz}} S^{\mathrm{iz}}-\phi_{\mathrm{Iz}} S^{\mathrm{Iz}}-\phi_{\mathrm{Dis}} S^{\mathrm{Dis}}
\end{aligned}
$$

and

$$
\begin{aligned}
S_{\mathrm{i}}^{p}= & \frac{3}{2} \frac{m_{\mathrm{i}}}{m_{\mathrm{n}}} T_{\mathrm{n}} S^{n}+\frac{1}{2} m_{\mathrm{i}}\left(\boldsymbol{u}_{\mathrm{n}}-\boldsymbol{u}_{\mathrm{i}}\right)^{2}\left(S^{n}+S^{\mathrm{cx}}\right) \\
& +\frac{1}{2} m_{\mathrm{i}} u_{\mathrm{i}}^{2} S^{n}+\left(\boldsymbol{u}_{\mathrm{n}}-\boldsymbol{u}_{\mathrm{i}}\right) \cdot \boldsymbol{R}_{\mathrm{in}}^{\mathrm{cx}}+Q_{\mathrm{in}}^{\mathrm{cx}}-Q_{\mathrm{ni}}^{\mathrm{cx}} .
\end{aligned}
$$

Here, $\boldsymbol{u}_{\mathrm{n}}$ is the neutral fluid velocity, which is zero in the fluid model presented in Sec. IIB but included here for future reference. The charge-exchange and dissociation source terms are

$$
\begin{gathered}
S^{\mathrm{cx}}=n_{\mathrm{i}} n_{\mathrm{warm}}\left\langle\sigma_{\mathrm{Iz}} v\right\rangle, \\
S^{\text {Dis }}=n_{\mathrm{e}} n_{\text {cold }}\left\langle\sigma_{\text {Dis }} v\right\rangle,
\end{gathered}
$$

where the reaction rates in (17), (22), and (23) can be found by parameterizations of polynomial fits to experimental data in terms of $T_{\mathrm{e}, \mathrm{i}}$ (see, for example, Ref. 27). The ion-neutral and neutral-ion frictional momentum transfer terms from charge exchange can be approximated by

$$
\begin{aligned}
\boldsymbol{R}_{\mathrm{in}}^{\mathrm{cx}} \approx & -m_{\mathrm{i}} \sigma_{\mathrm{cx}}\left(v_{\mathrm{cx}}\right) n_{\mathrm{i}} n_{\mathrm{warm}}\left(\boldsymbol{u}_{\mathrm{i}}-\boldsymbol{u}_{\mathrm{n}}\right) \\
& \times \frac{v_{\mathrm{th}, \mathrm{n}}^{2}}{\sqrt{\frac{16}{\pi} v_{\mathrm{th}, \mathrm{i}}^{2}+4\left(\boldsymbol{u}_{\mathrm{i}}-\boldsymbol{u}_{\mathrm{n}}\right)^{2}+\frac{9 \pi}{4} v_{\mathrm{th}, \mathrm{n}}^{2}}}, \\
\boldsymbol{R}_{\mathrm{ni}}^{\mathrm{cx}} \approx & m_{\mathrm{i}} \sigma_{\mathrm{cx}}\left(v_{\mathrm{cx}}\right) n_{\mathrm{i}} n_{\mathrm{warm}}\left(\boldsymbol{u}_{\mathrm{i}}-\boldsymbol{u}_{\mathrm{n}}\right) \\
& \times \frac{v_{\mathrm{th}, \mathrm{i}}^{2}}{\sqrt{\frac{16}{\pi} v_{\mathrm{th}, \mathrm{n}}^{2}+4\left(\boldsymbol{u}_{\mathrm{i}}-\boldsymbol{u}_{\mathrm{n}}\right)^{2}+\frac{9 \pi}{4} v_{\mathrm{th}, \mathrm{i}}^{2}}}
\end{aligned}
$$

where $v_{\mathrm{cx}}=\sqrt{\frac{4}{\pi}\left(v_{\mathrm{th}, \mathrm{i}}^{2}+v_{\mathrm{th}, \mathrm{n}}^{2}\right)+\left(\boldsymbol{u}_{\mathrm{i}}-\boldsymbol{u}_{\mathrm{n}}\right)^{2}}$, and the heatexchange from charge-exchange collision can be approximated by

$$
\begin{aligned}
Q_{\mathrm{in}}^{\mathrm{cx}} \approx & \frac{3}{4} m_{\mathrm{i}} \sigma_{\mathrm{cx}}\left(v_{\mathrm{cx}}\right) n_{\mathrm{i}} n_{\mathrm{warm}} v_{\mathrm{th}, \mathrm{n}}^{2} \\
& \times \sqrt{\frac{4}{\pi} v_{\mathrm{th}, \mathrm{i}}^{2}+\left(\boldsymbol{u}_{\mathrm{i}}-\boldsymbol{u}_{\mathrm{n}}\right)^{2}+\frac{64}{9 \pi} v_{\mathrm{th}, \mathrm{n}}^{2}}, \\
Q_{\mathrm{ni}}^{\mathrm{cx}} \approx & \frac{3}{4} m_{\mathrm{i}} \sigma_{\mathrm{cx}}\left(v_{\mathrm{cx}}\right) n_{\mathrm{i}} n_{\mathrm{warm}} v_{\mathrm{th}, \mathrm{i}}^{2} \\
& \times \sqrt{\frac{4}{\pi} v_{\mathrm{th}, \mathrm{n}}^{2}+\left(\boldsymbol{u}_{\mathrm{i}}-\boldsymbol{u}_{\mathrm{n}}\right)^{2}+\frac{64}{9 \pi} v_{\mathrm{th}, \mathrm{i}}^{2}},
\end{aligned}
$$

following Refs. 13 and 28.

We will now show how the source terms enter the drift fluid equations. Following the recipe of drift-ordering, the 
momentum source terms, i.e., Eqs. (18) and (19), introduce additional drifts. The drift ordering used here is identical to that of Refs. 8 and 18 and allows for solving the momentum equations iteratively, by assuming that the characteristic time- and parallel length-scales are much larger the inverse ion gyro-frequency and the ion gyro-radius. This allows for describing the perpendicular momentum vector (including terms up to first order) by

$$
\boldsymbol{u}_{\perp \sigma}=\boldsymbol{u}_{\perp \sigma}^{0}+\boldsymbol{u}_{\perp \sigma}^{1},
$$

where $\boldsymbol{u}_{\perp \sigma}^{0}$ are the (leading-order) diamagnetic- and $E \times B$ drifts

$$
\boldsymbol{u}_{\perp \sigma}^{0}=-\frac{\nabla_{\perp} p_{\sigma} \times \hat{\boldsymbol{B}}}{q_{\sigma} n B}-\frac{\nabla_{\perp} \phi \times \hat{\boldsymbol{B}}}{B},
$$

and $\boldsymbol{u}_{\perp \sigma}^{1}$ consist of the (next-to-leading-order) polarization, resistive, and viscous drifts

$$
\boldsymbol{u}_{\perp \sigma}^{1}=\boldsymbol{u}_{\mathrm{p} \sigma}+\boldsymbol{u}_{\mathrm{R} \sigma}+\boldsymbol{u}_{\pi \sigma} .
$$

Similar to the friction between ions and electrons, the neutral sources also originate from collisions at a much lower frequency than that of the ion gyration and thus introduce a first order drift $\boldsymbol{u}_{\mathrm{S} \sigma}$ to the existing sum in (30), resulting in

$$
\boldsymbol{u}_{\perp \sigma}^{1}=\boldsymbol{u}_{\mathrm{p} \sigma}+\boldsymbol{u}_{\mathrm{R} \sigma}+\boldsymbol{u}_{\pi \sigma}+\boldsymbol{u}_{\mathrm{S} \sigma} .
$$

Since the source term itself is first order in the driftapproximation, it is only the leading order velocity terms that contribute, i.e.,

$$
\boldsymbol{u}_{\mathrm{S} \sigma}=\frac{\boldsymbol{S}_{\sigma}^{u} \times \hat{\boldsymbol{B}}}{n_{\sigma} q_{\sigma} B},
$$

where

$$
\begin{gathered}
\boldsymbol{S}_{\mathrm{e}}^{u}=m_{\mathrm{e}}\left(\boldsymbol{u}_{\mathrm{n}}-\boldsymbol{u}_{\perp \mathrm{e}}^{0}\right) S^{n}, \\
\boldsymbol{S}_{\mathrm{i}}^{u}=m_{\mathrm{i}}\left(\boldsymbol{u}_{\mathrm{n}}-\boldsymbol{u}_{\perp \mathrm{i}}^{0}\right)\left(S^{n}+S^{\mathrm{cx}}\right)+\boldsymbol{R}_{\mathrm{in}}^{\mathrm{cx}}-\boldsymbol{R}_{\mathrm{ni}}^{\mathrm{cx}} .
\end{gathered}
$$

This results in the source drifts

$$
\begin{gathered}
\boldsymbol{u}_{\mathrm{Se}}=\frac{S^{n}}{n_{\mathrm{e}} \Omega_{\mathrm{ce}}}\left[\boldsymbol{u}_{\perp \mathrm{n}}-\left(\frac{\nabla_{\perp} \phi}{B}+\frac{\nabla_{\perp} p_{\mathrm{e}}}{q_{\mathrm{e}} n_{\mathrm{e}} B}\right)\right] \\
\boldsymbol{u}_{\mathrm{Si}}=\frac{S^{n}+S^{\mathrm{cx}}}{n_{\mathrm{i}} \Omega_{\mathrm{ci}}}\left[\boldsymbol{u}_{\perp \mathrm{n}}-\left(\frac{\nabla_{\perp} \phi}{B}+\frac{\nabla_{\perp} p_{\mathrm{i}}}{q_{\mathrm{i}} n_{\mathrm{i}} B}\right)\right]+\frac{\boldsymbol{R}_{\perp \mathrm{in}}^{\mathrm{cx}}-\boldsymbol{R}_{\perp \mathrm{ni}}^{\mathrm{cx}}}{n_{\mathrm{i}} \Omega_{\mathrm{ci}}} .
\end{gathered}
$$

Had elastic collisions with neutrals been included in the model, a yet another first order drift term would be added to (31). This is the so-called Pedersen drift, and details on the derivation of the term are found in Ref. 8.

In Sec. II D, the new source-drift terms are introduced in the HESEL drift-fluid based model for the plasma edge and SOL regions.

\section{Combined model equations}

The source terms from neutral interactions that were calculated in Sec. II C enter the HESEL equations as

$$
\begin{gathered}
\mathrm{d}_{t} n+n \mathcal{K}(\phi)-\mathcal{K}\left(p_{\mathrm{e}}\right)=\Lambda_{n}+\Sigma_{n} \\
\nabla \cdot\left(\mathrm{d}_{t}^{0} \nabla_{\perp} \phi^{*}\right)-\mathcal{K}\left(p_{\mathrm{e}}+p_{\mathrm{i}}\right)=\Lambda_{\omega}+\Sigma_{\omega} \\
\frac{3}{2} \mathrm{~d}_{t} p_{\mathrm{e}}+\frac{5}{2} p_{e} \mathcal{K}(\phi)-\frac{5}{2} \mathcal{K}\left(p_{\mathrm{e}}^{2} / n\right)=\Lambda_{p_{\mathrm{e}}}+\Sigma_{p_{\mathrm{e}}} \\
\frac{3}{2} \mathrm{~d}_{t} p_{\mathrm{i}}+\frac{5}{2} p_{i} \mathcal{K}(\phi)+\frac{5}{2} \mathcal{K}\left(p_{\mathrm{i}}^{2} / n\right)-p_{\mathrm{i}} \mathcal{K}\left(p_{\mathrm{e}}+p_{\mathrm{i}}\right)=\Lambda_{p_{\mathrm{i}}}+\Sigma_{p_{\mathrm{i}}},
\end{gathered}
$$

where

$$
\begin{gathered}
\Sigma_{n}=S_{\mathrm{e}}^{n}-\nabla \cdot\left(n \boldsymbol{u}_{\mathrm{e}}^{\mathrm{S}}\right), \\
\Sigma_{\omega}=\left(S_{\mathrm{i}}^{n}-S_{\mathrm{e}}^{n}\right)-\nabla \cdot\left[n\left(\boldsymbol{u}_{\mathrm{i}}^{\mathrm{S}}-\boldsymbol{u}_{\mathrm{e}}^{\mathrm{S}}\right)\right], \\
\Sigma_{p_{\mathrm{e}}}=S_{\mathrm{e}}^{p}-\frac{3}{2} \nabla \cdot\left(p_{\mathrm{e}} \boldsymbol{u}_{\mathrm{e}}^{\mathrm{S}}\right)-p_{\mathrm{e}} \nabla \cdot \boldsymbol{u}_{\mathrm{e}}^{\mathrm{S}}, \\
\Sigma_{p_{\mathrm{i}}}=S_{\mathrm{i}}^{p}-\frac{3}{2} \nabla \cdot\left(p_{\mathrm{i}} \boldsymbol{u}_{\mathrm{i}}^{\mathrm{S}}\right)-p_{\mathrm{i}} \nabla \cdot \boldsymbol{u}_{\mathrm{i}}^{\mathrm{S}} .
\end{gathered}
$$

The neutrals are described as three fluids, consisting of cold thermal molecules, warm atoms from dissociation of molecules, and hot atoms from charge-exchange collisions with ions. The transport of neutral particles is described by diffusion (8) with source terms (10)-(12). The diffusion coefficients are approximated by

$$
D_{\text {hot }}=10^{1} D_{\text {warm }}=10^{3} D_{\text {cold }}=10^{2} \rho_{\mathrm{s}}^{2} \Omega_{\text {ci }},
$$

which have approximately the same relative value as prescribed by (9).

Note that the neutral terms have been normalized with parameters that are related to the plasma parameters and not to those of the neutrals. If one were to treat only the neutral particles, more suitable scale-setting parameters may exist, but for the combined model, it is simpler to apply the same normalization throughout. The system of equations is solved in a slab geometry in a drift-plane at the outboard mid-plane of a tokamak, perpendicular to the magnetic field lines. The domain is depicted in Fig. 2, which has dimensions of 225 $\times 75 \rho_{\mathrm{s}}^{2}$ and consists of SOL and edge sub-domains.

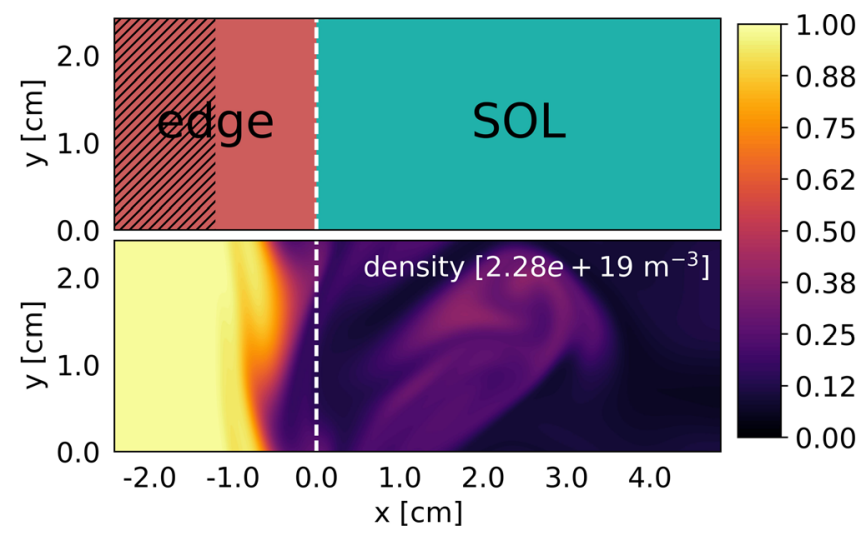

FIG. 2. The upper frame shows the domain for the combined HESELneutral simulations, where the $x$-coordinate corresponds to the radial direction and the $y$-coordinate corresponds to the poloidal direction. In the inner part of the edge region, marked by the shaded area, the plasma density and pressure fields are relaxed towards fixed prescribed profiles, which result in a dynamically imposed boundary condition to the core. In the SOL region, the plasma fields are damped according to parametrized parallel transport. The white dashed line shows the location of the LCFS. The lower frame displays a snapshot of the turbulent electron density during a simulation. 
The boundaries are periodic in the poloidal $y$-direction. At the inner boundary towards the core, the boundary conditions for the plasma fields are $\omega=0, n=1.5 n_{0}$, and $p_{\mathrm{e}}=2 p_{\mathrm{i}}$ $=10 n_{0} T_{\mathrm{e} 0}$, and at the outer boundary towards the first wall, the boundary conditions are $\partial_{x} n=\partial_{x} p_{\mathrm{e}, \mathrm{i}}=\omega=0$, where $x$ is the radial direction. The neutral density fields satisfy

$$
-D_{\mathrm{n}} \partial_{x} n_{\mathrm{n}}=\sqrt{-\frac{D_{\mathrm{n}} S_{\mathrm{n}}^{\prime}}{n_{\mathrm{n}}}} n_{\mathrm{n}},
$$

at the inner boundary, where $S_{\mathrm{n}}^{\prime}$ are the source terms that are proportional to $n_{\mathrm{n}}$, i.e., for $\mathrm{n}=$ warm, the proportional source term is

$$
S_{\text {warm }}^{\prime}=-n_{\text {warm }}\left(n_{\mathrm{e}}\left\langle\sigma_{\mathrm{iz}} v\right\rangle+n_{\mathrm{i}}\left\langle\sigma_{\mathrm{cx}} v\right\rangle\right) .
$$

At the outer boundary, the neutral density boundary conditions are

$$
-D_{\mathrm{n}} \partial_{x} n_{\mathrm{n}}=-\gamma \sqrt{-\frac{D_{\mathrm{n}} S_{\mathrm{n}}^{\prime}}{n_{\mathrm{n}}}} n_{\mathrm{n}}+\Gamma_{\text {puff }} \delta_{\mathrm{n}, \text { cold }} .
$$

The boundary conditions in both (46) and (48) are obtained by solving (8) at a steady state, under the assumption that there are no fluctuations on the boundaries and that the density vanishes at $\pm \infty$. The outer boundary condition (48) moreover has a factor $\gamma$, which is a measure of the fraction of absorbed particles. For $\gamma=1$, the boundary conditions for the neutrals are (apart from a sign) identical to those on the inner boundary, i.e., solutions to the corresponding steady-state equations. If $\gamma=0$, no particles pass the boundary, and the outer boundary becomes a zero-flux boundary condition for the neutral atom densities. For the simulations used for this paper, the value is $\gamma=0.2$. The parameter $\Gamma_{\text {puff }}$ introduces a flux of thermal molecules to the system to mimic gas-puffing in a tokamak.

In Sec. III, the above model is solved in the BOUT ++ framework $^{29}$ for varying input parameters for the purpose of enlightening phenomena involving interactions between neutral particles and edge and SOL plasmas.

\section{APPLICATIONS}

The inclusion of neutral interactions in the HESEL driftfluid model equations allows for investigating various aspects of the edge and SOL fusion plasmas. In this section, it is illustrated how gas-puff rates affect the far edge and SOL profiles for electron density and for electron and ion temperatures. It is moreover shown that for an appropriate flux of neutrals into the system, the density source in the edge region can be replaced by a self-consistent source from ionization of neutral particles. For the results presented here, the gyro-Bohm normalization parameters from (5) correspond to the plasma parameters of a medium-sized tokamak with

$$
\begin{array}{ll}
\Omega_{\mathrm{ci}}=9.57 \times 10^{7} \mathrm{~s}^{-1}, & T_{\mathrm{e} 0}=20 \mathrm{eV}, \\
\rho_{\mathrm{s}}=3.23 \times 10^{-4} \mathrm{~m}, & T_{\mathrm{i} 0}=10 \mathrm{eV}, \\
n_{0}=1.5 \times 10^{19} \mathrm{~m}^{-3}, & B_{0}=2 \mathrm{~T},
\end{array}
$$

and with deuterium ions and neutrals.

\section{A. Changing neutral molecule puffing rate}

The model described in Sec. II D is solved numerically with the same initial plasma fields but for different values of flux of neutral molecules into the system at the outer boundary. The purpose is to investigate how different gas-puff rates affect the plasma density and temperature profiles and turbulent dynamics in the edge and SOL regions.

The values of the flux density $\Gamma_{\text {puff }}$ are $10^{20} \mathrm{~m}^{-2} \mathrm{~s}^{-1}$ resembling a low puffing rate, $10^{21} \mathrm{~m}^{-2} \mathrm{~s}^{-1}$ for a medium puffing rate, and $10^{22} \mathrm{~m}^{-2} \mathrm{~s}^{-1}$ for a high puffing rate. The low and medium puffing rates resemble realistic puffing rates for experiments. For all puffing rates, the plasma density and pressure sources in the edge region are maintained, and the effect of the puffing rate on the profiles is thus only visible in the far edge and SOL regions.

It is observed from Fig. 3 that a higher puffing results in a higher average density and lower average electron and ion temperatures. This is particularly visible for the high puffing rate case.

Other profiles of interest are the electron/ion density source profiles, which are shown in Fig. 4. The density source profile provides the total ionization rate of atoms and molecules as a function of the radial position and can thus be used to estimate the location of the fuelling. There is a clear tendency in the density source profiles for an increased gas puffing to increase the magnitude of the ionization source as expected from (17). It is moreover observed that for an increased gas puffing, the profile peaks in both the edge and SOL move towards the LCFS. Relatively more neutrals are ionized in the SOL for the lower fuelling cases, and we believe that the reason for this is that the higher fuelling cases cool the outermost electrons more, which allows for a deeper penetration of the neutral molecules before they are ionized. In Fig. 5, the radial electric field is shown. The radial electric field is known to correlate with the formation of an edge transport barrier due to the sheared poloidal flow

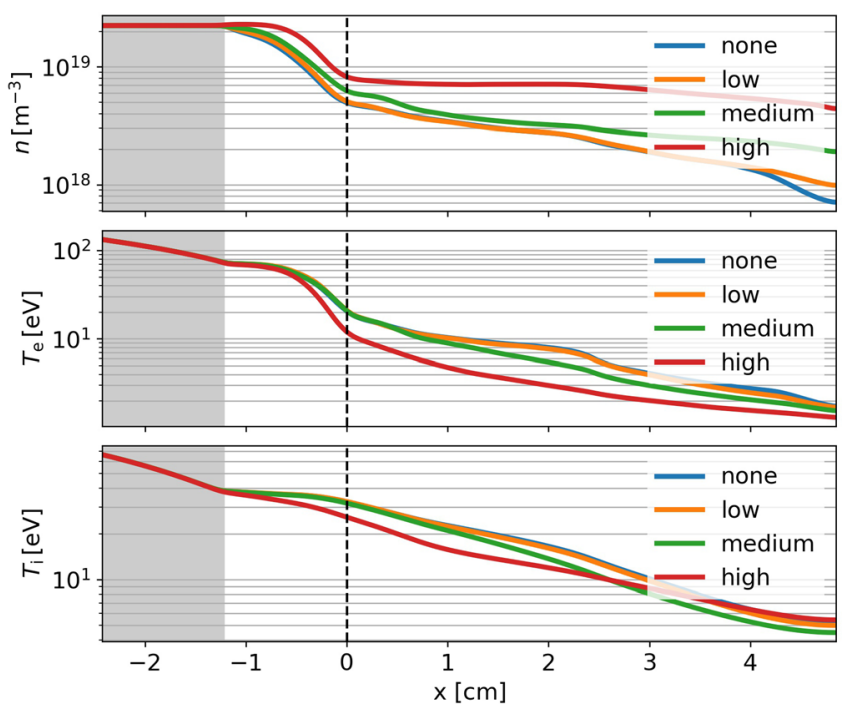

FIG. 3. Temporally averaged electron density and ion and electron temperature profiles for "none," "low," "medium," and "high" gas puffing rates. Neutral gas puffing increases the density and decreases the temperatures in the edge and SOL regions. The gray region marks the forcing region. 


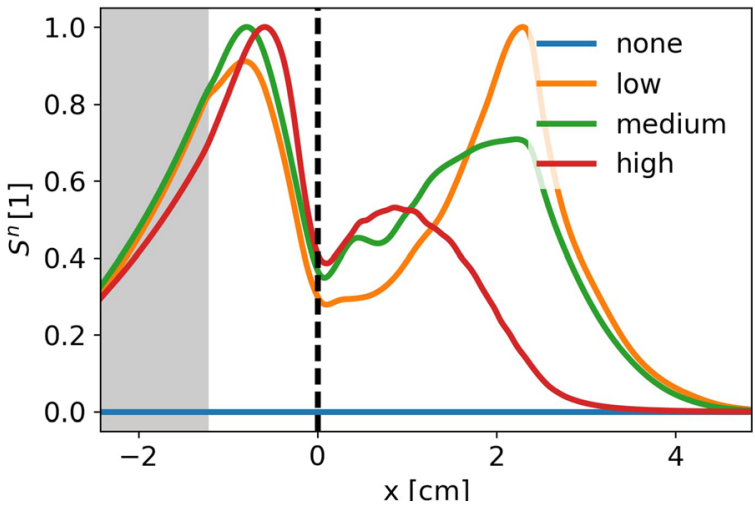

FIG. 4. The normalized density source for "none," "low," "medium," and "high" gas puffing rates. The profiles are normalized with the values $1,1.41$ $\times 10^{21}, 1.33 \times 10^{22}$, and $1.18 \times 10^{23}$, respectively, all in units of $\mathrm{m}^{-3} \mathrm{~s}^{-1}$. The density source increases with the amount of neutrals, and the source in the SOL in particular is shifted towards the LCFS for higher fuelling rates. The gray region marks the forcing region.

resulting from the $E \times B$-drift. A negative radial electric field corresponds to an $E \times B$ flow in the electron-diamagnetic velocity direction, which is usually observed in experiments. It is observed that the radial electric field changes slightly with the puffing rate, where in particular for the case of high gas puffing, the profile of the radial electric field shifts away from that where neutrals are absent.

The plasma edge dynamics also change when the gas puffing is increased. This can be observed by monitoring the particle flux across the LCFS. Among the leading order drifts, only the $E \times B$-drift advects the density, and the radial particle flux $\Gamma_{x}$ is thus obtained from

$$
\Gamma_{x}=-n \frac{\partial_{y} \phi}{B} .
$$

From Fig. 6, it appears that there is a tendency for the average radial density flux to increase with a higher puffing rate, which is consistent with the increased fuelling in the edge region. The flux characteristics are also different for different cases, which is apparent in Fig. 7 showing the probability density functions (PDFs) for $\Gamma_{x}$. The mean, standard deviation, skewness, and kurtosis for the PDFs are shown in

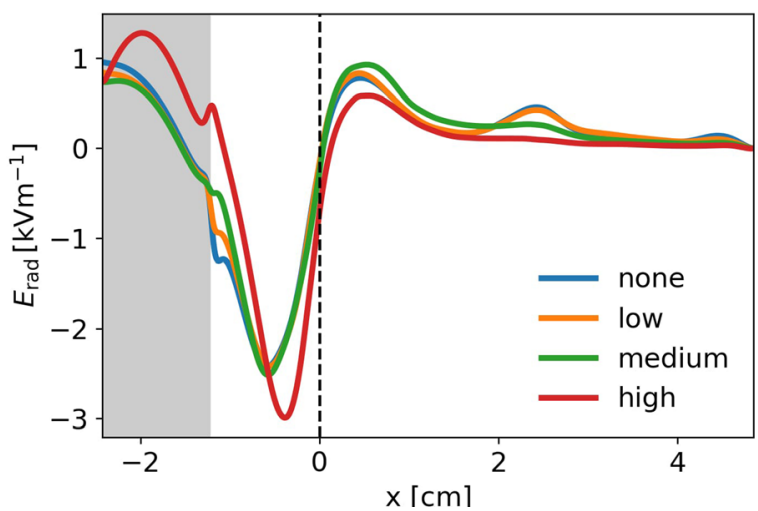

FIG. 5. The radial electric field for "none," "low," "medium," and "high" gas puffing rates. It is observed that the radial electric field changes slightly both in the magnitude and shape with increased neutral flux. The gray region marks the forcing region.
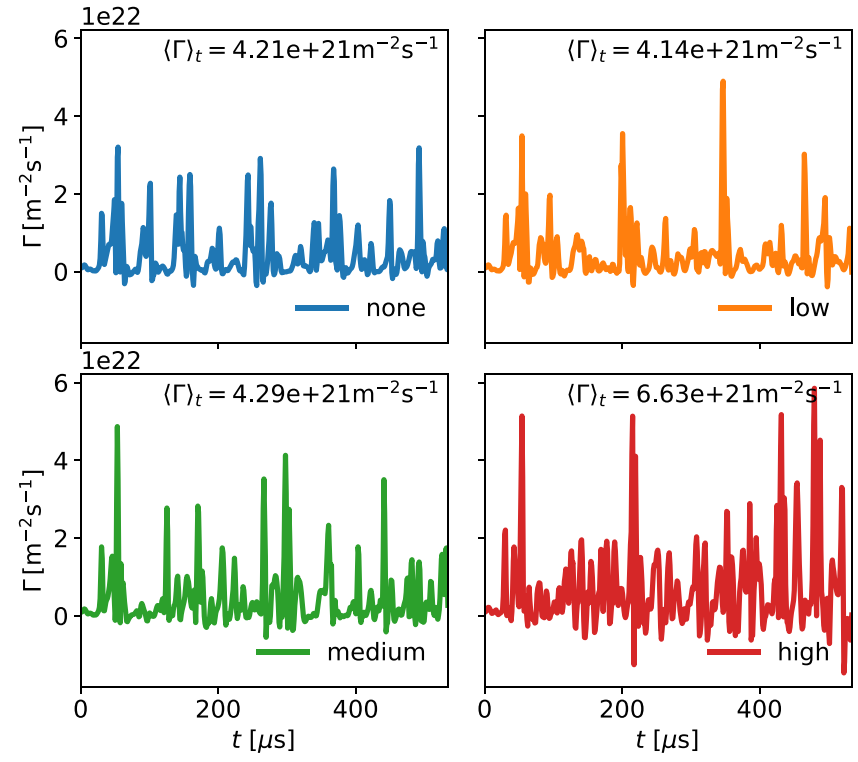

FIG. 6. Radial particle flux across LCFS for "none," "low," "medium," and "high" gas puffing rates. The general tendency is that the flux increases with the increased puffing rate, and the characteristics of the transport do likewise appear to be influenced by the neutral density.

Table I. For all gas puff cases, except for that with no puffing, there is a trend that the mean and standard deviation increase, and the skewness and kurtosis decrease, for a higher puffing rate. It is unclear why the values for the simulation without gas puffing deviate so much from that with low puffing, but it is our belief that larger datasets will show a better consistency between the statistics of the flux for different puffing rates. A more detailed analysis on the effect of the fuelling rate on blob characteristics is planned for a future publication. The topic is related to the results in Ref. 30 , where the effect of neutrals on blob transport is attributed to their insulating behavior in the divertor region. It is interesting that we see similar effects but without any inclusion of physics near the divertor. Future studies with the model should likewise enlighten whether the rise of density in the SOL observed in Fig. 3 is mainly due to increased ionization in this region or a larger radial particle flux across the LCFS as a result of increased fuelling.

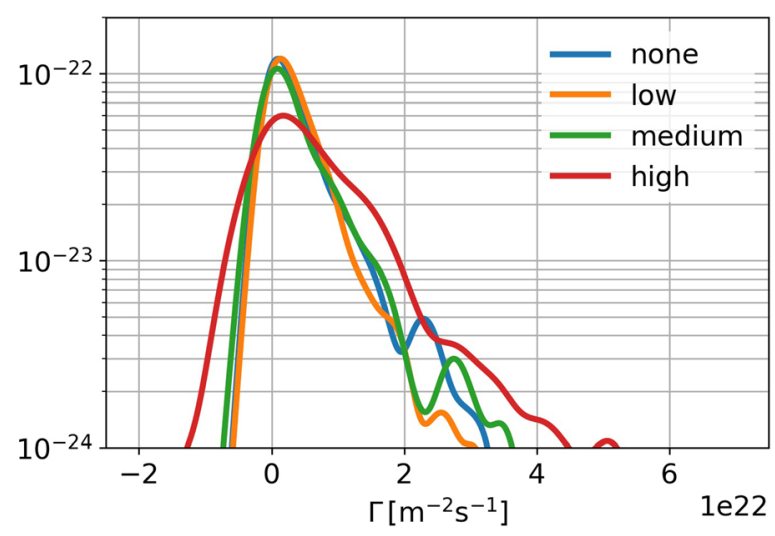

FIG. 7. Probability density of the flux across LCFS for "none," "low," "medium," and "high" gas puffing rates. Descriptors for the PDFs are given in Fig. 1. 


\section{B. The effect of the puffing rate on self-consistent fuelling}

In this section, we investigate the possibility of removing the forcing of the density that is responsible for the stiffness in the innermost part of the domain, as shown by the shaded area in Fig. 3. The forcing has been necessary in previous plasma simulations since the profiles are naturally flattened due to transport. Here, the density source is supplemented by a self-consistent source from local ionization of neutrals, and the forcing at the inner boundary acts as a source (or as a sink) to constitute an appropriate coupling towards the core plasma.

With the inclusion of the density source from local ionization of neutrals, it is, however, possible to remove the forcing altogether. Removing the forcing is desirable since for tokamak experiments, fuelling is entirely caused by ionization of neutrals. It is unknown whether the fuelling from gas puffing is approximately evenly distributed across the plasma surface, which would correspond to removing the profile forcing as done here. Alternatively, more neutrals may cross the LCFS locally, for example, near the x-point after recycling at the divertor, and the resulting plasma is then transported upstream. The fuelling from particles recycled in the simulated domain is also not included although those may contribute with a significant amount. ${ }^{5}$ Neutrals originating from recycling can be included in the model by introducing a term for the atomic species similar to that for the flux of molecules on the outer boundary in (48) or by increasing the value of $\Gamma_{\text {puff }}$, which would then represent a sum of flux from puffed and recycled neutral molecules.

For the simulations treated in Sec. III A, both the integrated forced density source, i.e.,

$$
\text { force }=\int_{\text {edge }} \mathrm{d} x \mathrm{~d} y\left(n_{\mathrm{fixed}}-n\right) / \tau
$$

where $n_{\text {fixed }}$ is the fixed density profile and $\tau$ is the relaxation time, and the integrated source from ionization of neutrals, i.e.,

$$
\text { fuel }=\int_{\text {edge }} \mathrm{d} x \mathrm{~d} y S^{n}
$$

are shown in Fig. 8. This should give a hint of the amount of gas puffing necessary for keeping the edge density up. It appears that the density source at low puffing is completely dominated by factors other than local ionization of neutrals,

TABLE I. Mean, standard deviation, skewness, and kurtosis for the PDF of the radial particle flux across the LCFS. The mean only changes significantly when going from medium to high fuelling, whereas the skewness and kurtosis appear to depend stronger on the gas puff rate.

\begin{tabular}{lcccc}
\hline \hline & $\begin{array}{c}\text { Mean } \\
\left(10^{21} \mathrm{~m}^{-2} \mathrm{~s}^{-1}\right)\end{array}$ & $\begin{array}{c}\text { Standard dev. } \\
\left(10^{21} \mathrm{~m}^{-2} \mathrm{~s}^{-1}\right)\end{array}$ & Skewness & Kurtosis \\
\hline None & 4.21 & 5.92 & 2.08 & 7.80 \\
Low & 4.14 & 5.95 & 3.29 & 18.9 \\
Medium & 4.29 & 6.87 & 2.51 & 11.4 \\
High & 6.63 & 9.92 & 1.92 & 8.25 \\
\hline \hline
\end{tabular}

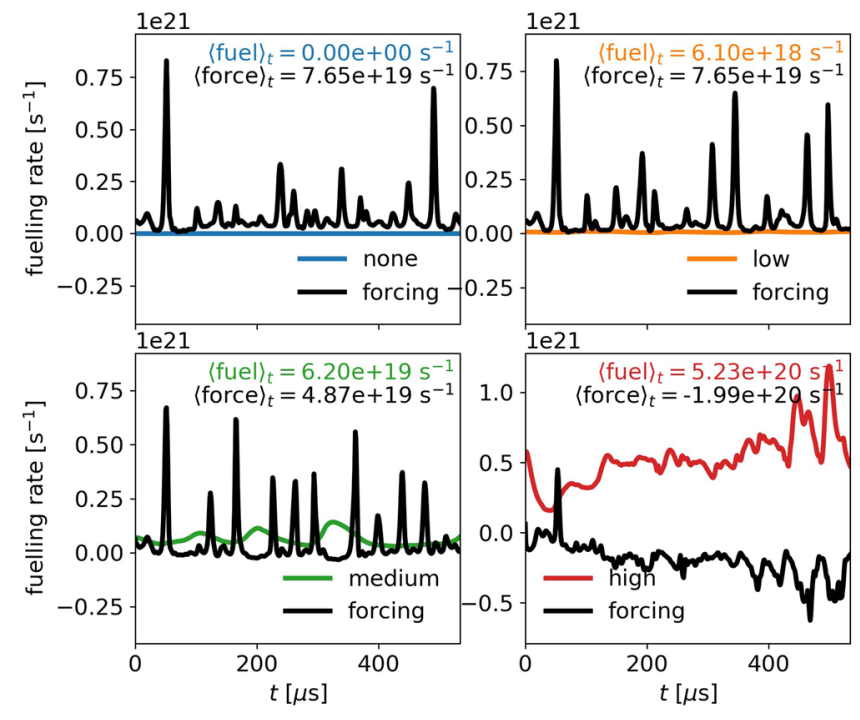

FIG. 8. Fuelling in the edge for "none," "low," "medium," and "high" gas puffing rates. The black curves show the forcing applied to maintain the density in the inner edge region and the colored curves the amount of fuelling provided by ionization of neutrals. For the high puffing rate, the neutrals fuel more than what the system is targeted for and the forcing becomes negative in order to maintain the density in the inner edge.

at medium puffing the two sources contribute approximately equally, and at a high puffing rate, the source from local ionization of neutrals is so big that the forcing becomes negative. Note that for the "none," "low," and "medium" cases where the neutrals do not fuel the plasma sufficiently, the blob events are visible as peaks in the forcing graph. This feature, however, ceases to exist when the neutrals fuel the plasma, and the forcing turns negative. The change in the characteristics of the "high" puffing plot in Fig. 8 from that of the other plots is thus due to a change in the degree of local fuelling from neutrals, going from under-fueled to over-fueled, and not due to a change in the blob formation mechanism itself.

The ideal puffing rate result in a vanishing average forcing, and from the above observations, this should be expected to lie somewhere in between the cases with the medium and high fuelling rate. It appears that with a puffing rate of $\Gamma_{\text {puff }}=2 \cdot 10^{21} \mathrm{~m}^{-2} \mathrm{~s}^{-1}$, the plasma edge density is maintained at approximately $n=1.5 n_{0}$, which allows for a scenario where the edge region in the simulation is entirely fueled from the local ionization of neutrals.

\section{CONCLUSIONS}

In Sec. II, it is displayed how inelastic source terms enter a drift-fluid model. It is found that density sources give rise to an additional drift term in the electron and ion drift velocities and that yet another term is added to the ion drift velocity due to charge-exchange collisions with neutrals.

Neutral atoms originating from dissociation of molecules, and to an even greater extend atoms that have undergone charge-exchange collisions with ions, experience a mean-free path between collisions in the SOL, which is on the order of meters and thus much larger than any gradient length-scales perpendicular to the magnetic field lines. For this reason, a neutral model that is valid in the long 
mean-free path limit has been formulated. The neutral model is coupled to the HESEL drift-fluid model for the plasma fields, and the resulting system of equations describes the evolution of plasma and neutral fields in a self-consistent manner.

The coupled HESEL-neutral model allow for investigation of various phenomena in Sec. III. It is observed how increasing the amount of neutrals raises the electron and ion densities and lowers the temperatures in the SOL and far edge regions. It is moreover observed that change in the flux of neutrals into the system affects the radial electric field and the statistical properties of the electron and ion particle fluxes across the LCFS. The self-consistent model moreover allows for removing the forcing of the density source in the inner edge region present in the original HESEL equations and introduces a source from local ionization of neutrals in the edge region.

A combined plasma-neutral model as the one described in this paper allows for a wide range of studies on the neutral-plasma interactions. It is possible to further illuminate how the interactions with neutrals affect blobs and how blobs affect neutrals, with a model that includes both dynamical neutrals and plasma fields. Moreover, a range of experimentally observed phenomena that are most likely due to neutrals influencing the plasma fields, such as shoulder formation $^{30}$ and the fuelling triggered H-mode density limit, ${ }^{31}$ are expected to at least be partially explained by future simulations with this model.

\section{ACKNOWLEDGMENTS}

This work was carried out within the framework of the EUROfusion Consortium and received funding from the Euratom research and training programme 2014-2018 under Grant Agreement No. 633053. The views and opinions expressed herein do not necessarily reflect those of the European Commission.

${ }^{1}$ S. J. Zweben, J. A. Boedo, O. Grulke, C. Hidalgo, B. LaBombard, R. J. Maqueda, P. Scarin, and J. L. Terry, Plasma Phys. Controlled Fusion 49, S1 (2007).

${ }^{2}$ O. E. Garcia, V. Naulin, A. H. Nielsen, and J. Juul Rasmussen, Phys. Plasmas 12, 62309 (2005).

${ }^{3}$ D. A. D'Ippolito1, J. R. Myra, and S. J. Zweben, Phys. Plasmas 18, 060501 (2011).
${ }^{4}$ V. Naulin, J. Nucl. Mater. 363-365, 24 (2007).

${ }^{5}$ U. Samm and the TEXTOR-94 Team, Plasma Phys. Controlled Fusion 41, B57 (1999).

${ }^{6}$ S. J. Zweben, J. L. Terry, D. P. Stotler, and R. J. Maqueda, Rev. Sci. Instrum. 88, 041101 (2017).

${ }^{7}$ E. de la Cal and The TJ-II Team, Nucl. Fusion 56, 106031 (2016).

${ }^{8}$ M. Løiten, Ph.D. thesis, Department of Physics, Technical University of Denmark, 2017.

${ }^{9}$ C. Wersal and P. Ricci, Nucl. Fusion 55, 123014 (2015).

${ }^{10}$ D. Reiter, M. Baelmans, and P. Börner, Fusion Sci. Technol. 47, 172 (2005).

${ }^{11}$ D. Heifetz, D. Post, M. Petravic, J. Weisheit, and G. Bateman, J. Comput. Phys. 46, 309 (1982).

${ }^{12}$ K. Shimizu, T. Takizuka, S. Sakurai, H. Tamai, H. Takenaga, H. Kubo, and Y. Miura, J. Nucl. Mater. 313-316, 1277 (2003).

${ }^{13}$ E. T. Meier and U. Shumlak, Phys. Plasmas 19, 072508 (2012).

${ }^{14}$ E. L. Vold, A. K. Prinja, F. Najmabadi, and R. W. Conn, J. Nucl. Mater. 176-177, 570 (1990).

${ }^{15}$ D. Schwörer, N. R. Walkden, H. Leggate, B. D. Dudson, F. Militello, T. Downes, and M. M. Turner, Nucl. Mater. Energy 12 825 (2017).

${ }^{16}$ A. S. Thrysøe, L. E. H. Tophøj, V. Naulin, J. Juul Rasmussen, J. Madsen, and A. H. Nielsen, Plasma Phys. Controlled Fusion 58, 044010 (2016).

${ }^{17}$ S. Chapman and T. G. Cowling, The Mathematical Theory of Non-uniform Gases (Cambridge, 1953).

${ }^{18}$ J. Madsen, V. Naulin, A. H. Nielsen, and J. Juul Rasmussen, Phys. Plasmas 23, 032306 (2016).

${ }^{19}$ A. H. Nielsen, J. Juul Rasmussen, J. Madsen, G. S. Xu, V. Naulin, J. M. B. Olsen, M. Løiten, S. K. Hansen, N. Yan, and L. Tophøj, Plasma Phys. Controlled Fusion 59, 025012 (2017).

${ }^{20}$ F. L. Hinton and C. W. Horton, Jr., Phys. Fluids 14, 116 (1971).

${ }^{21}$ A. Zeiler, J. F. Drake, and B. Rogers, Phys. Plasmas 4, 2134 (1997).

${ }^{22}$ C. Cercignani, The Boltzmann Equation and Its Applications (Springer, New York, NY, 1988).

${ }^{23}$ P. C. Stangeby, The Plasma Boundary of Magnetic Fusion Devices (Institute of Physics Publishing, Bristol, 2000).

${ }^{24}$ R. D. Hazeltine, Phys. Plasmas 5, 3282 (1998).

${ }^{25}$ J. D. Hey, C. C. Chu, P. Mertens, S. Brezinsek, and B. Unterberg, J. Phys. B: At. Mol. Opt. Phys. 37, 2543-2567 (2004).

${ }^{26} \mathrm{P}$. Mertens and A. Pospieszczyk, J. Nucl. Mater. 266-269, 884-889 (1999).

${ }^{27}$ R. K. Janev, W. D. Langer, K. Evans, Jr., and D. E. Post, Jr., Elementary Processes in Hydrogen-Helium Plasmas (Springer-Verlag, Berlin, 1987).

${ }^{28}$ H. L. Pauls, G. P. Zank, and L. L. Williams, J. Geophys. Res. 100, 21595, https://doi.org/10.1029/95JA02023 (1995).

${ }^{29}$ B. D. Dudson, M. V. Umansky, X. Q. Xu, P. B. Snyder, and H. R. Wilson, Comput. Phys. Commun. 180, 1467 (2009).

${ }^{30}$ D. Carralero, J. Madsen, S. A. Artenea, M. Bernert, G. Birkenmeier, T. Eich, G. Fuchert, F. Laggner, V. Naulin, P. Manz et al., Nucl. Mater. Energy 12, 1189 (2017).

${ }^{31}$ M. Bernert, T. Eich, A. Kallenbach, D. Carralero, A. Huber, P. T. Lang, S. Potzel, F. Reimold, J. Schweinzer, E. Viezzer, and H. Zohm, Plasma Phys. Controlled Fusion 57, 014038 (2015). 